\title{
TOPOGRAPHIC ARRANGEMENT OF CRYSTALLITE ORIENTATIONS IN ROLLED COPPER
}

\author{
R. GOTTHARDT, G. HOSCHEK, O. REIMOLD, and F. HAESSNER \\ Max-Planck-Institut für Metallforschung, Institut für Sondermetalle, Stuttgart, Germany
}

(Received January 25, 1972, in final form June 8, 1972)

\begin{abstract}
In single and polycrystalline copper rolled to a reduction of $95 \%$, the topographic arrangements of crystallite orientations were determined over large areas by means of electron microscopy. Correlated data on orientation, diffraction site, and transmission pictures in three cross-sections perpendicular to the normal, rolling and transverse directions were obtained and evaluated. These results were complemented by X-ray studies of the texture. The rolled polycrystalline structure consists of roughly disc-shaped regions of uniform orientation which are fairly strongly misoriented with respect to each other. The planes of the discs lie parallel to the rolling plane. Within these regions of uniform orientation lie elongated subgrains extended in the longitudinal and transverse directions. In a surface layer of a few microns thickness the dryly rolled single crystals have essentially a (100)[011] texture. This is independent of the orientation of the interior of the material. The observed structures are discussed with respect to possible formation mechanisms.
\end{abstract}

\section{INTRODUCTION}

The description of the structure of a rolled metal is a complex problem. In a first approximation, this problem consists of the determination of the size, shape and arrangement of the crystallites and of their orientation distribution. $\dagger$ In a second approximation the description should also contain corresponding information on the lattice defects.

In recent years the orientation distribution of a large number of crystallites has been determined and analysed for heavily rolled copper, brass and niobium ${ }^{1-8}$ by means of selected-area diffraction (SAD-technique) in electron microscopy. In addition, the three-dimensional orientation distribution function for some cubic metals was determined from quantitative pole figures for various lattice planes (X-ray-technique) by an analysis involving an expansion into a series of generalized spherical harmonics. ${ }^{9-11}$ In the case of rolled copper, the three-dimensional orientation distribution function has been established from electron microscopic data of individual orientations as well as from $\mathrm{X}$-ray pole figure data by the method of generalized spherical harmonics. ${ }^{7}$ Thus there exists to-day with the exception of some subtle details which are still

\footnotetext{
$\dagger$ The significance and the justification of the use of the term "crystallite" in the case of a strongly deformed metal are not a priori clear. The word crystallite is used in the same sense as, for example, in the numerous theories of rolling textures which consider the "rotation of crystallites" into certain orientations.
}

open to discussion, a quantitative and mathematically exact description of the complicated copper texture which was disputed for a long time. There is little doubt that the two independent methods, i.e. SAD and X-ray techniques, can be applied to many materials in order to determine the primary set of data necessary for the mathematical analysis.

On the other hand, the determination of the other fundamental parameters of the crystallite structure is still in its infancy. However, as we have shown earlier, ${ }^{1,12}$ the SAD technique permits not only the study of the orientations of the crystallites, but also of the above-mentioned other parameters of the crystallite structure, i.e. the mutual orientation relationship (orientation topography). However, in order to make a correct statement concerning the spatial arrangement of the crystallites in a rolled metal, it is necessary to investigate the material in three mutually orthogonal sections. ${ }^{12,13}$

In connection with problems of nucleation, $\mathrm{Hu}$ has investigated by electron microscopy the fine structure of small material regions in cross-sections of rolled copper sheets. ${ }^{14,15}$ Results on larger regions are not available so far. The present investigation provides information of this kind on heavily rolled poly- and single-crystalline copper. The data were obtained by means of electron microscopy with the combined assignment of orientation, diffraction site, and transmission micrograph. The resulting possible consequences concerning the structure of the rolled material will be discussed. 


\section{EXPERIMENTAL PROCEDURE}

A piece of copper $99.999+\%$ pure was repeatedly forged, rolled and annealed. A texture-free strip, $1 \mathrm{~mm}$ thick, having a largely uniform recrystallized structure with grain sizes between 10 and $15 \mu \mathrm{m}$ was obtained as the starting material. This strip was then cold-rolled reversely to a reduction of $95 \%$. In addition, single crystals of the same copper having the dimensions of $50 \times 50 \times 4 \mathrm{~mm}$ were grown in high-purity graphite moulds by directional solidification from the melt. The orientations of these single crystals agreed within $2^{\circ}$ with the seeded orientations (123)[745]], (112)[645] and (123) [121] $]$. These specimens were also cold-rolled reversely to a reduction of $95 \%$. All the rolled samples were stored in liquid nitrogen until the next treatment.

For the electron microscopic observations thin foils were prepared from the rolled strips parallel to three principal cross-sections of the material: the rolling plane, the plane perpendicular to the rolling direction (transverse cross-section) and the plane perpendicular to the transverse direction (longitudinal cross-section). The preparation of the two latter sections was made possible by electroplating the rolled strip with copper before sectioning. ${ }^{14-16}$ Thinning was done in the usual manner. In order to perform X-ray studies of the rolling plane, different layers of the material were exposed by chemically polishing one side of the specimens.

Information on the determination of orientation distributions in rolled metals by the electron microscopic SAD-technique was given in an earlier publication ${ }^{1}$ and in more detail recently. ${ }^{17} \dagger$ By determining the orientations of diffraction sites distributed in a grid-like arrangement, the mutual local arrangements can also be evaluated by this technique (orientation topography). ${ }^{1,12}$ The information of such an orientation topography depends of course on the chosen grid spacing and on the diameter of the diffraction site contributing to the diffraction pattern.

Since our investigations were aimed primarily at obtaining information on the orientation topography of larger areas of material, a grid spacing as

$\dagger$ The ambiguity which occurs for certain singular orientations in indexing the electron microscopic diffraction patterns ${ }^{18}$ is not a serious limitation of the method for fcc metals if, as was done in the present work, zone axes of sufficiently high indices are employed in the evaluation. ${ }^{17}$ Under these conditions, the probability of occurrence of the above-mentioned singular orientations becomes negligibly small. large as possible was desirable. On the other hand, a too large grid spacing leads to the loss of too many details. For these reasons, an optimum value was established empirically. Preliminary experiments yielded a useful grid spacing of $1 \mu \mathrm{m}$. In the case of the polycrystalline material, it was necessary to use a spacing of $0.5 \mu \mathrm{m}$ in the direction of the sheet normal. The SAD pattern corresponded to a region of material of about $0.2 \mu \mathrm{m}$ diameter. The selection of significantly smaller regions is limited by the increasing inaccuracy in the determination of the position of the investigated site. ${ }^{19}$

In order to reduce the contamination of the foil in the electron microscope, a cooling stage was employed in the objective chamber, and the diffraction patterns were photographed from outside with a camera. ${ }^{20}$ This permitted the observation time to be extended, so that more than 200 consecutive diffraction patterns could be photographed, when foils with large transparent regions were used.

In addition to the electron microscopic orientation determinations (111)- and (200)-pole figures were obtained from a series of specimens by conventional X-ray diffractometer studies using a counter tube. ${ }^{21,22}$ Filtered $\mathrm{Co}-\mathrm{K}_{\alpha}$ or $\mathrm{Cr}-\mathrm{K}_{\alpha}$ radiation was used. The contours in the pole figures are either stated in relative units or, where necessary, normalized with respect to statistical units.

\section{EXPERIMENTAL RESULTS AND THEIR EVALUATION}

\section{Polycrystalline Material}

Topography of the rolling plane In total, about 900 diffraction patterns were photographed from 14 different areas of the material. In addition, one transmission electron micrograph was taken from each area. The following results are based on grids corresponding to the two largest connected specimen areas with 100 and 238 diffraction sites. These will subsequently be referred to as series I and II, respectively. All other diffraction series were used to substantiate statistically the results of series I and II, such as, for example, the absence of certain orientations.

In order to illustrate the characteristic features of the orientation distribution in a (111)-pole figure, the results of 16 grid elements of series I are shown as an example in Figure 1. Corresponding (111)-poles were marked with the same grid element 


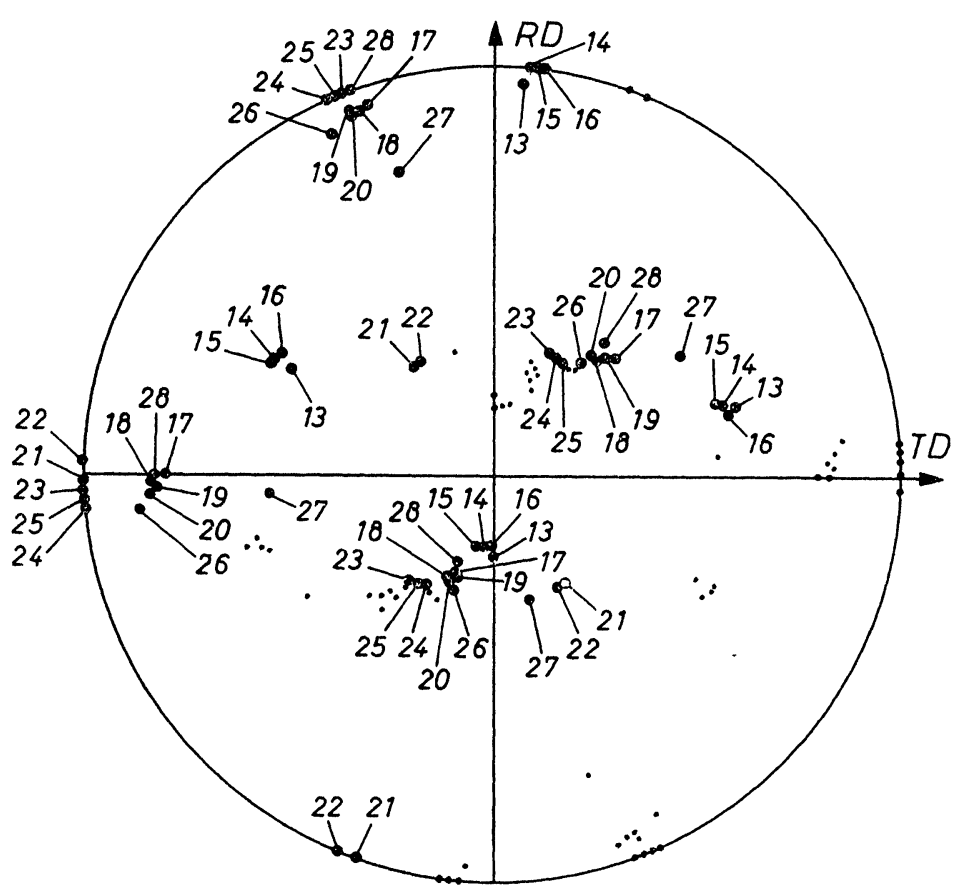

FIGURE 1 Stereographic projection showing orientations of 16 grid sites arranged in a square grid. (111)-poles; the poles of the point-symmetric orientations are not labelled with grid element numbers).

number. $\uparrow$ The grid element numbers indicate which sites of the foil had the corresponding orientations. Even this small number of orientations allows one to recognize clearly a predominance of certain orientations (e.g. the groups $13,14,15,16$ or 17,18 , $19,20)$. Since the evaluation of the orientations was accurate within about $5^{\circ}, 1,17$ such ranges of preferential orientation scattered over less than $10^{\circ}$ permit the conclusion that "uniformly" oriented regions existed in the foil. These ranges of preferential orientation will be characterized by their mean orientations which will be referred to as mean preferential orientations in the following sections. Orientations were only considered as part of such ranges of preferential orientation, if all four (111)poles were compatible with the mean preferential orientations within the individual ranges of scatter. Orientations that could not be assigned to any of the preferential orientations were considered as stray orientations. However, these cases occurred

$\dagger$ Since orientations can only be determined from the electron microscopic diffraction patterns within an uncertainty of a rotation of $180^{\circ}$ about the foil normal (ND), two orientations characterized by four (111)-poles, respectively belong to each diffraction site. The poles of the pointsymmetric orientations were not labelled with grid element numbers of clarity. relatively seldom, and no relation to the mean preferential orientations of the larger areas was apparent. For this reason, all stray orientations were

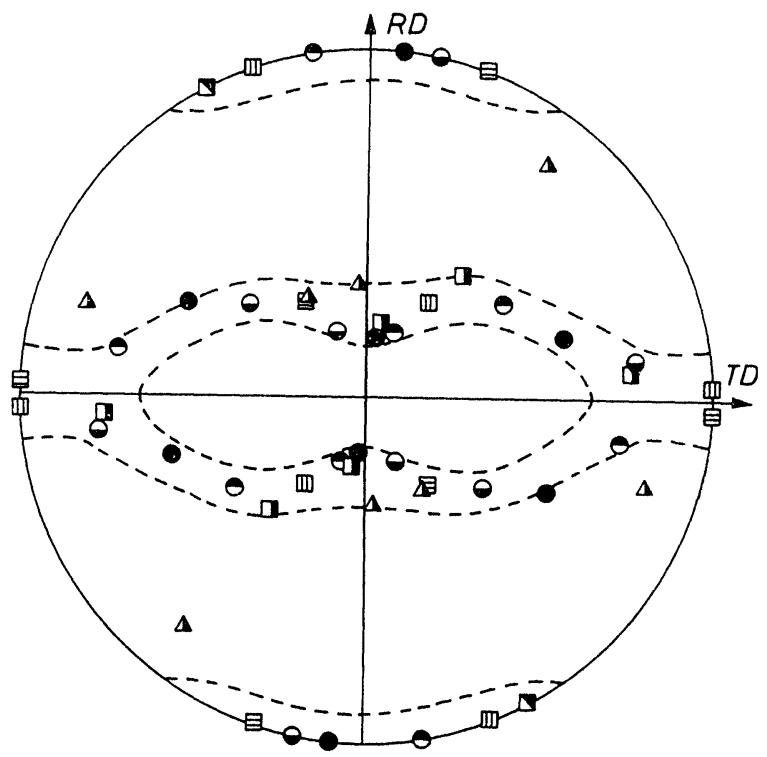

FIGURE 2 Stereographic projection showing (111)-poles of the mean preferential orientations of the areas of uniform orientation. Series I (compare Figure 3). 


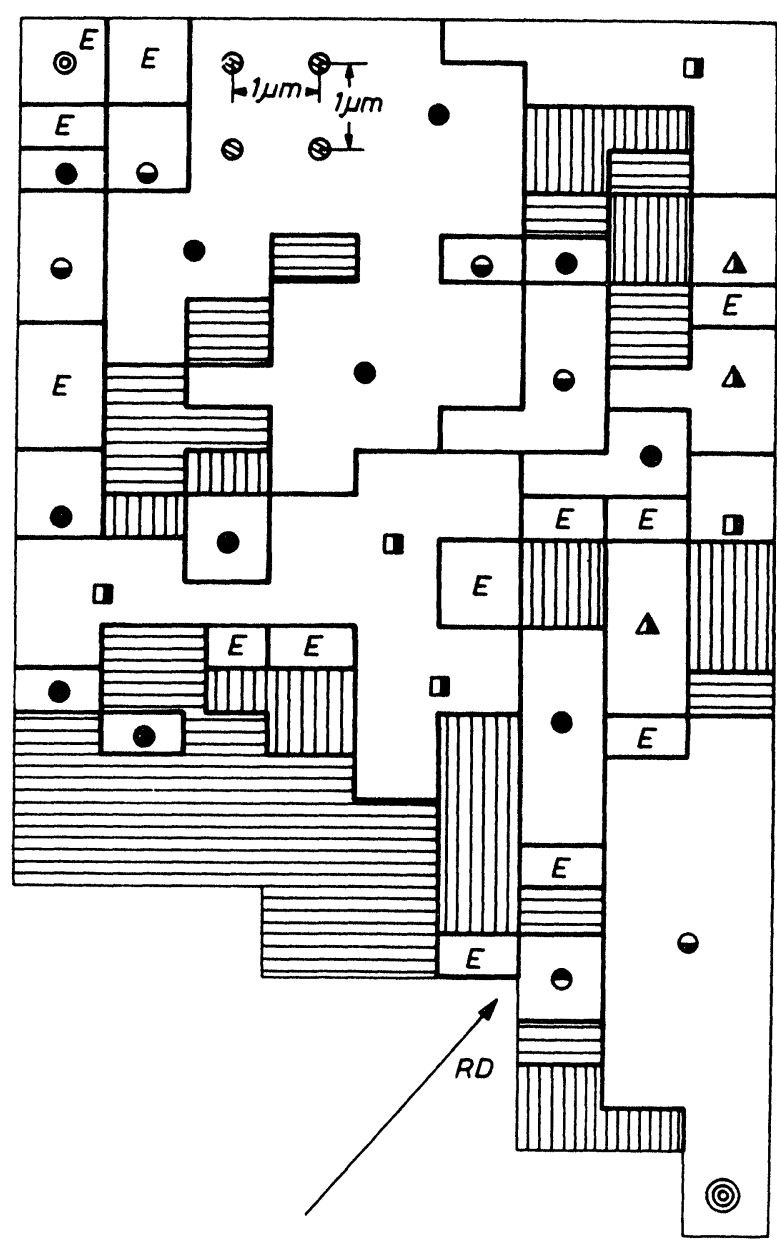

FIGURE 3 Topographic arrangement of the mean preferential orientations in the rolling plane. Series I (meaning of the symbols as in Figure 2; grid site).

labelled uniformly as E-orientations in the topographic representation, although these orientations differed individually.

In Figure 2 all mean preferential orientations which were found in series $I$ are represented in a (111)-pole figure. Each mean preferential orientation (and the corresponding point-symmetric position) within a series is characterized by a different symbol. The dashed lines represent the contours corresponding to a statistical unit, as determined by $\mathrm{X}$-rays. It is evident that the majority of the mean preferential orientations falls within this range of values of the texture of rolled copper. Finally Figure 3 shows the topographic arrangement of the mean preferential orientations of series I. The trans-

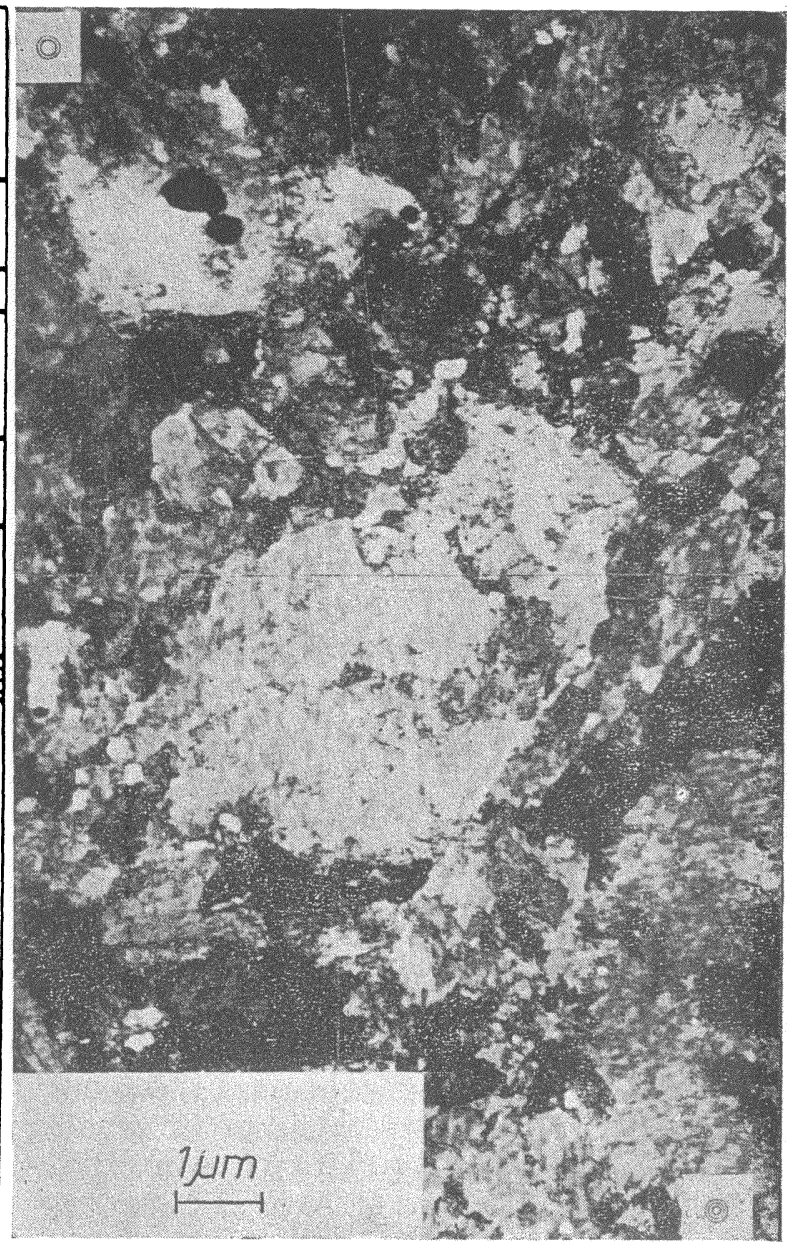

FIGURE 4 Transmission micrograph of the same region of material as in Figure 3 (same scale as in Figure 3; the sites labelled by $\odot$ resp. $\because$ in Figures 3 and 4 correspond to each other).

mission electron micrograph of this region of materials is shown in Figure 4 on the same scale as Figure $3 . \dagger$ The four regularly arranged dashed circles in Figure 3 represent some locations of the grid elements. The symbols of the encircled regions are the same as those characterizing the mean preferential orientations in Figure 2. Thus the mutual position of the mean preferential orientations can be determined easily with the aid of Figures 2 and 3.

It is clear that the major part of the grid consists of more or less large areas of uniform orientation.

$\uparrow$ The data of series II corresponding to Figures 2, 3 and 4 are quite similar and have hence been omitted. 
The boundaries of these regions cannot be determined by mere inspection of the transmission micrograph. Occasionally the regions are separated locally by regions of another orientation. However, since Figure 3 reflects only the two-dimensional arrangement in the foil, it could well be that, in a

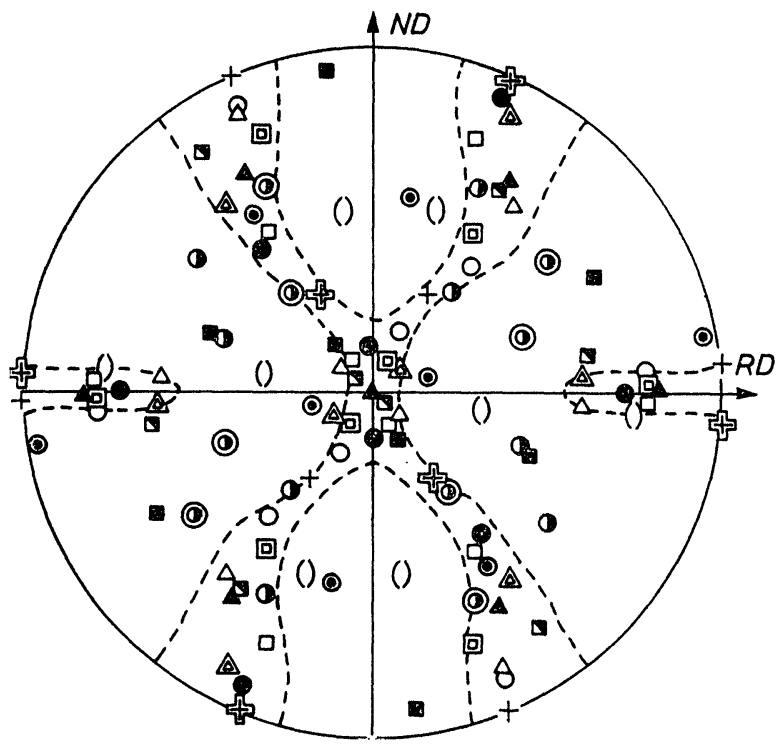

FIGURE 5 (111)-poles of the mean preferential orientations of the areas of uniform orientation in stereographic projection; investigated region of material as in Figure 7.

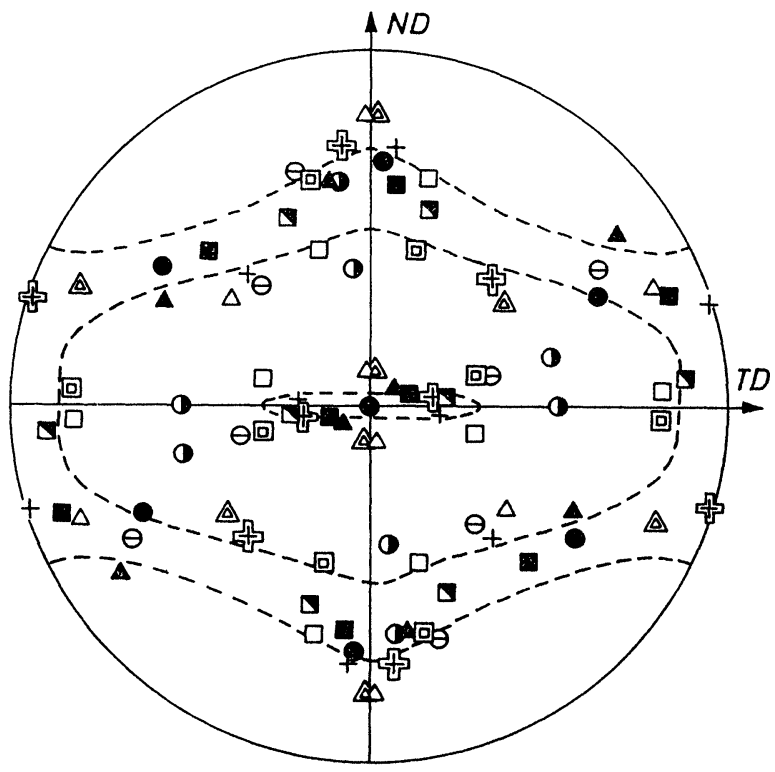

FIGURE 6 (111)-poles of the mean preferential orientations of the areas of uniform orientation in stereographic projection; investigated region of material as in Figure 8.
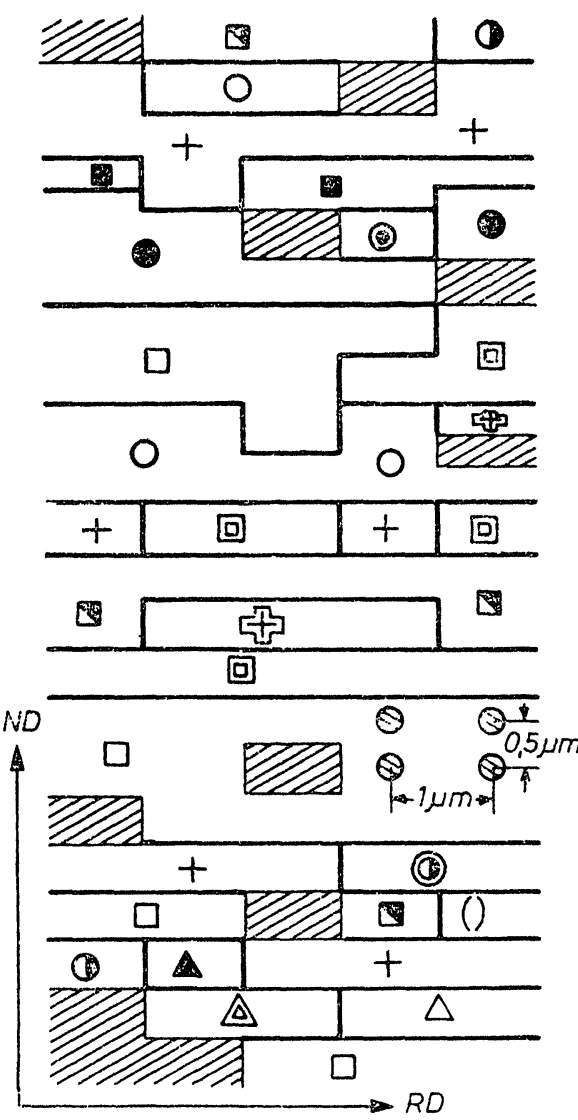

FIGURE 7 Topographic arrangement of the mean preferential orientations of the plane ND-RD (meaning of the symbols as in Figure 5; shaded regions are not evaluated; - grid site).

three-dimensional representation, the large regions are mutually interconnected by links. The topographic results of the other principal sections (see Figure 7 and Figure 8) do not exclude such an interpretation.

Some of the mean preferential orientations can be transformed into one another by reflection at the plane ND-RD (compare, for example, orientations $\ominus$ and $\ominus$ or $\||\||$ and $\equiv$ in Figure 2). Such "complementary" orientations occurred almost always in closely neighbouring regions of the foil and had common boundaries over more or less extended distances (this can be recognized particularly clearly in Figure 3 for the regions having the mean preferential orientations ||||| and $\equiv$ ).

Although connected areas of up to a total of $25 \times 13 \mu \mathrm{m}^{2}$ were investigated, it is difficult to describe the mean size of the regions of uniform orientation, since the size of the regions covered by 


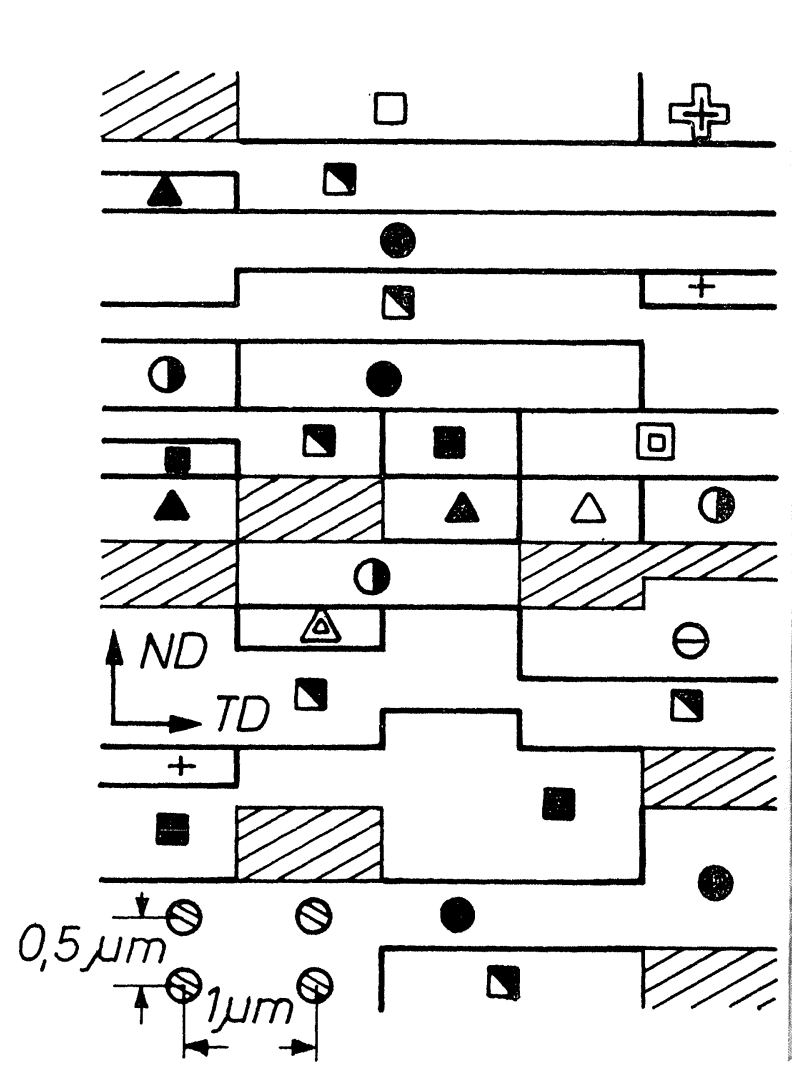

FIGURE 8 Topographic arrangement of the mean preferential orientations of the plane ND-TD (meaning of the symbols as in Figure 6; shaded regions are not evaluated; grid site).

the grid varied between 1 and $5 \mu \mathrm{m}$. Another disturbing feature of the topography in the rolling plane is that the shapes of the areas of uniform orientation show no indication of the rolling direction.

Topography of the planes $N D-R D$ and $N D-T D$ About 700 diffraction patterns corresponding to 21 different regions of material were analyzed. The largest connected grids contained 110 and 75 diffraction sites in the planes ND-RD and ND-TD respectively.

The observed orientations are concentrated around certain orientations in quite the same manner as described earlier. Figures 5 and 6 show these mean preferential orientations together with the point-symmetric positions for the two largest grids in the planes ND-RD and ND-TD in two (111)-pole figures. They are located mainly within the dashed range of scatter of the rolling texture of copper. The topographic arrangements of these

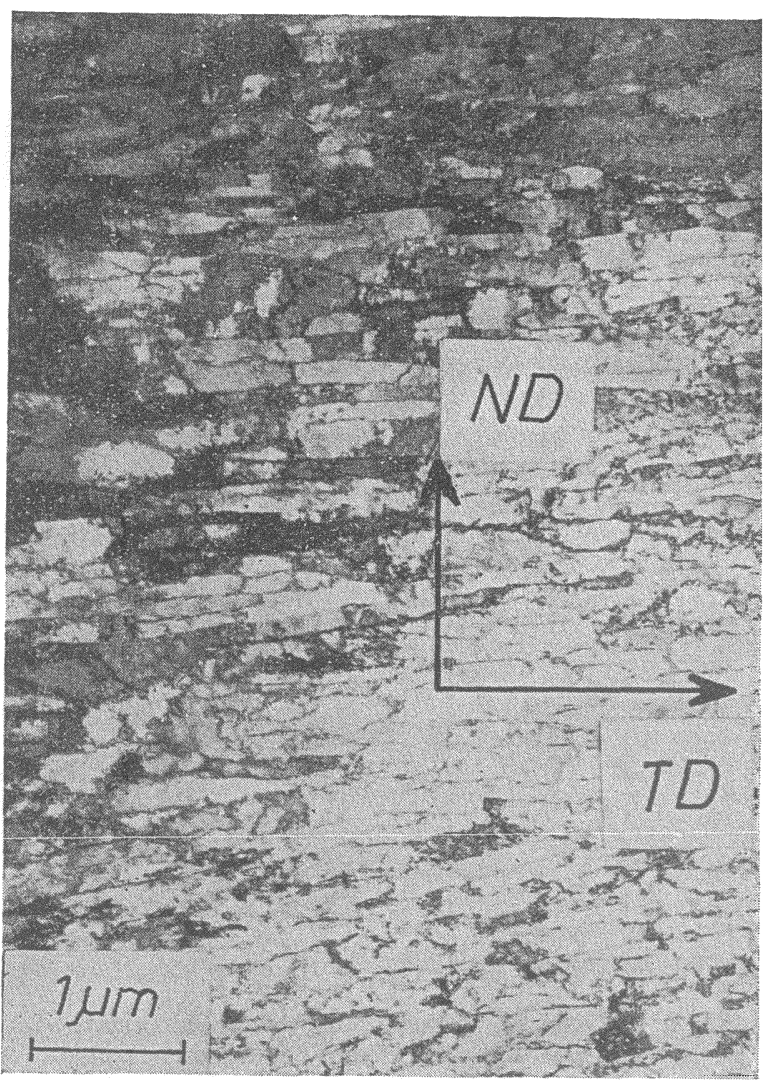

FIGURE 9 Transmission picture of the same region of material as in Figure 8 (same scale as in Figure 8).

mean preferential orientations are shown in Figures 7 and 8. The transmission electron micrographs of these two regions of material are practically identical. As an example, the transmission micrograph corresponding to the topographic arrangement of Figure 8 is shown in Figure 9. The topographic arrangements shown in Figures 7 and 8 extend right up to the rolling surface. $\dagger$ The positions of the mean preferential orientations near the surface correspond to Figures 5 and 6 . X-ray examinations also yjelded the same texture in the rolling surface as in the central plane of the strip.

The orientation topography of the longitudinal and the transverse cross-sections shows elongated cells of uniform orientations parallel to the rolling

$\dagger$ The surface could be determined very accurately on the transmission electron micrographs, since the appearance of the electrodeposited copper layer (very fine cells, extended perpendicularly to the surface and interspersed with twin lamellae) was entirely different from that of the rolled strip. 
and the transverse directions respectively. The shape of these regions is obviously similar in both crosssections. The thickness of the cells parallel to ND is about $0.5-1 \mu \mathrm{m}$; their length in RD and TD varies between 1 and $10 \mu \mathrm{m}$.

With our electron microscope only foils of less than about $0.1 \mu \mathrm{m}$ thick could be examined in transmission. It is therefore not surprising that these details are not revealed by the topographic picture of the rolling plane. By combining the topographic results in the three perpendicular crosssections, the following crude picture of the arrangement of the regions of uniform orientation emerges: The average region has the shape of a flat disc parallel to the rolling plane. Whereas the diameter of the disc varies considerably between 1 and $10 \mu \mathrm{m}$, the thickness is about $0.5-1 \mu \mathrm{m}$.

The transmission pictures also show elongated cells. There is, however, a difference between the structural significance of the cell boundaries in the transmission pictures and of the boundaries in the topographic representation. This difference will be considered in more detail in the section concerning the results on single crystals and in the discussion.

\section{Single-Crystalline Material}

Textures determined by X-ray techniques After rolling to a reduction of $95 \%$, the textures of the three single crystals of different orientations are

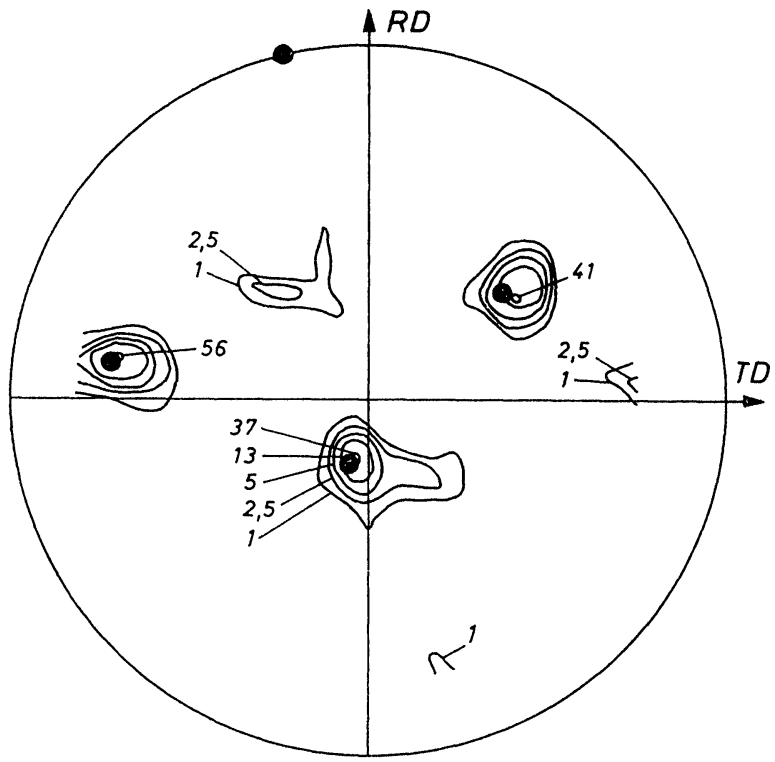

FIGURE 10 (111)-pole figure of a $95 \%$ rolled single crystal having initial orientation (123)[745] (back reflection region; intensities in relative units; $O$ : initial orientation). quite similar in the central plane of the rolled strips, cf. Figure 10. However, whereas the two crystals having initial orientations (123)[745] and (112)[645] exhibit hardly any change in orientation

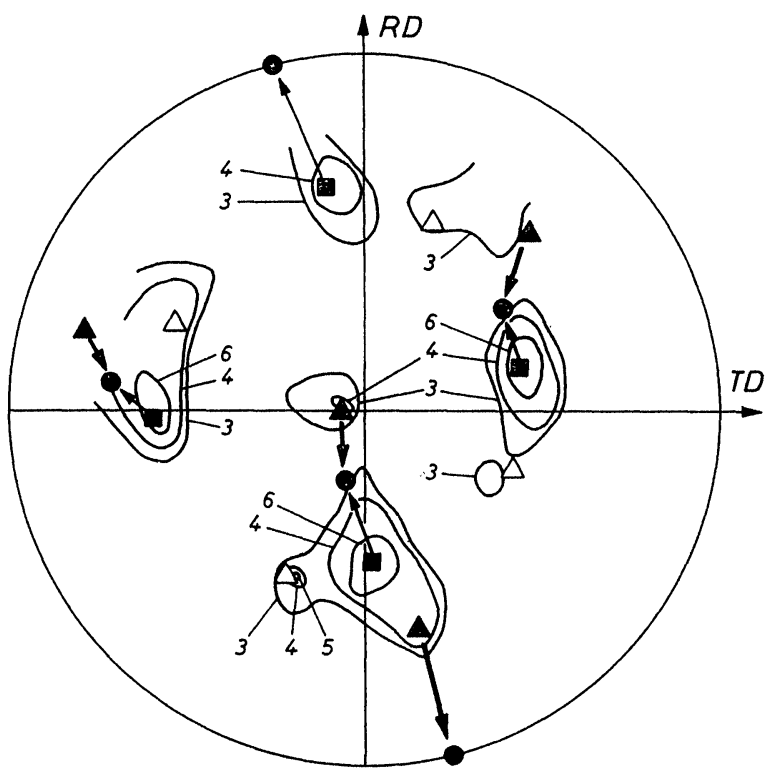

(a)

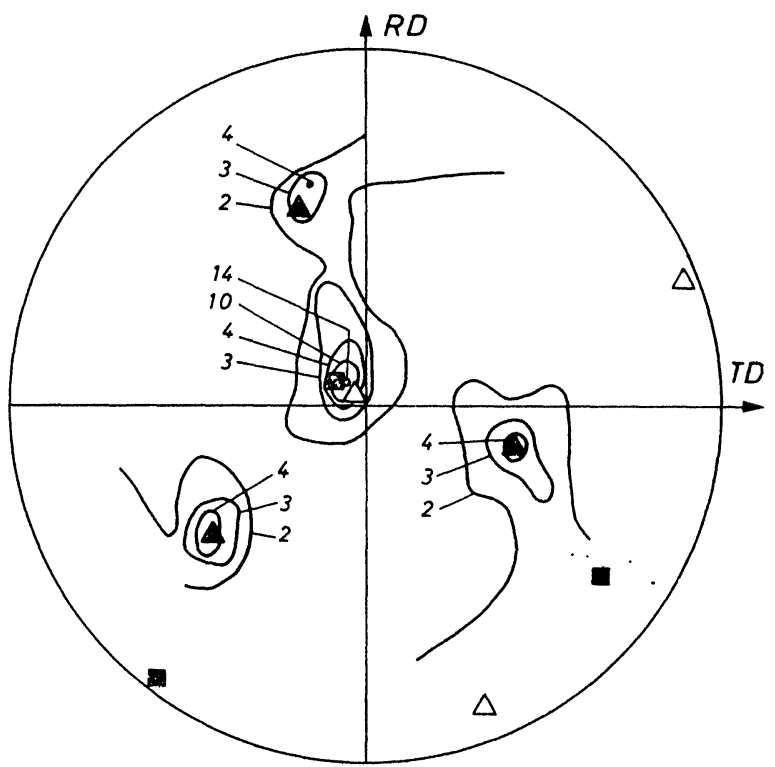

(b)

FIGURE 11 Surface texture of a $95 \%$ dryly rolled single crystal having initial orientation (123)[745] (a) (111)-pole figure (back reflection region; intensities in relative units; 口, $\Delta, \triangle$ : components $\mathrm{A}, \mathrm{B}, \mathrm{C}$; texture maxima of the interior of the material) (b) (200)-pole figure (back reflection region; intensities in relative units; $\mathbf{D}, \Delta, \Delta$ : components A, B, C). 
upon rolling, the crystal of initial orientation (123) [ī21] has experienced an extensive reorientation. Despite this dissimilar behaviour in the central plane, all three specimens develop essentially the same texture on the surface, namely (100)[011], provided rolling was performed without lubrication.

With $\mathrm{Cr}-\mathrm{K}_{\alpha}$ radiation, the depth of penetration in copper is only about $3-4 \mu \mathrm{m}$. This permits the study of surface texture in more detail. In the case of the (123)[745] crystals, the texture apparently consists of three components, as shown by the pole figures in Figures 11a and $\mathrm{b}$ : component $\mathrm{A}$, which
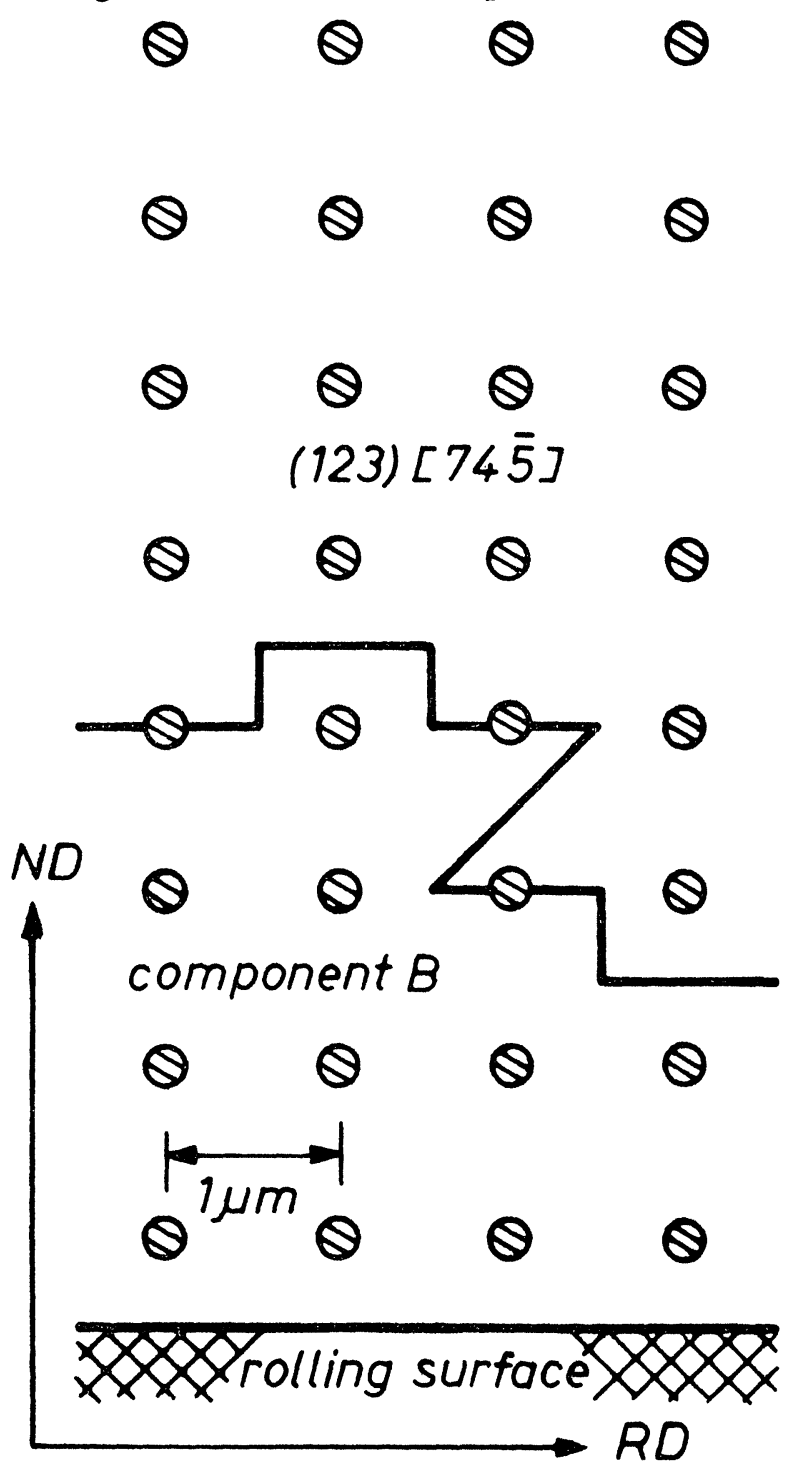

(a) resembles the (100)[011] texture, and components $B$ and $C$. Their relative frequency is about $8: 2: 1$. Upon removing a surface layer of a few microns, the only texture left to be observed corresponds to the texture of the central plane of the rolled strip.

\section{Topography of the planes $N D-R D$ and $N D-T D$}

These investigations were only performed on the (123) [745] ] crystals.

At a sufficient distance from the rolling surface, only the orientation (123)[745] is observed. In the vicinity of the surface, the component $A$ or the component $B$ occurs in addition. The surface texture component $\mathrm{C}$, which is very weak according to the X-ray measurements, could not be detected by electron microscopy.

Figures $12 \mathrm{a}$ and $\mathrm{b}$ show the topographic arrangement of component B. Accordingly, the surface orientation prevails to a depth of 3-4 $\mu \mathrm{m}$. Occasionally, diffraction patterns show spots of both the component $\mathrm{B}$ and the matrix orientation (cf. Figure
8
8
8
8
$\theta$
8
$\theta$
8
$\theta$
8
(123) [74 $\overline{5}]$

\begin{tabular}{|c|c|c|c|c|}
\hline$\theta$ & $\theta$ & $\theta$ & $\theta$ & $\theta$ \\
\hline$\theta$ & $\theta$ & $\theta$ & $\theta$ & $\theta$ \\
\hline
\end{tabular}

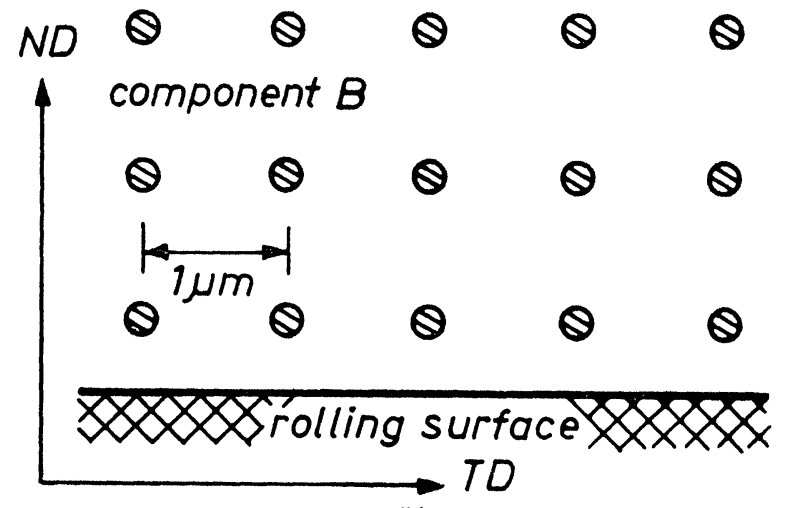

(b)

FIGURE 12 Topographic arrangement of the surface component B; $95 \%$ dryly rolled rolled single crystal having initial orientation (123) $[74 \overline{5}]$ ( grid sites) (a) investigated plane ND-RD (b) investigated plane ND-TD. 
12a). Such pictures permit the possible conclusion that layers of transitional orientations between the component $B$ and the (123)[745] matrix are so narrow that the corresponding diffraction spots are too weak to be detected. Despite this strong orientation gradient, the contrast of such regions on transmission micrographs shows no indication of this discontinuity. Instead, the same narrow, elongated cells which are characteristic of the heavily rolled polycrystalline material (cf. Figure 9) are observed near the surface and in the interior of the material (Figure 13). However, the cells are apparently larger in the immediate vicinity of the surface than they are deep in the interior.

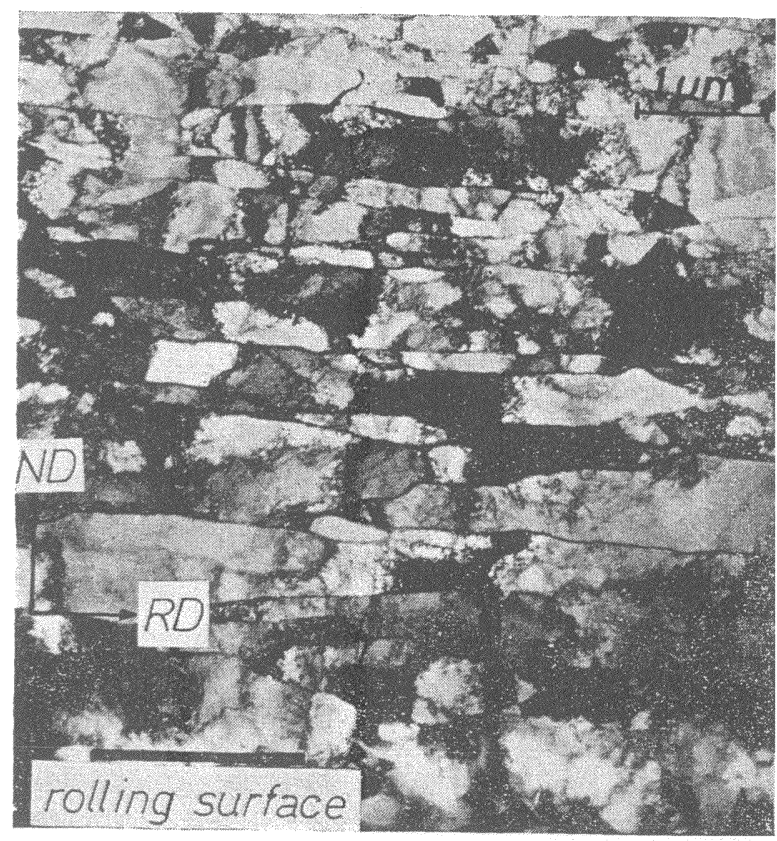

FIGURE 13 Transmission micrograph of the investigate region of material as in Figure 12a.

In contrast to the surface component $B$, the orientation of component A rotates continuously with increasing distance from the surface into the orientation (123)[745] , as shown in Figure 14. The positions labelled 1,2 and 3 correspond to the mean orientations, determined over a length of $4 \mu \mathrm{m}$ in the direction of $\mathrm{RD}$, at distances of 0.5 , 1.5 and $2.5 \mu \mathrm{m}$ from the surface respectively. At greater depths, no measurable change in the mean orientation was observed, it being close to the (123) [745] orientation of the matrix. This transition is not revealed by the contrast of the transmission images.

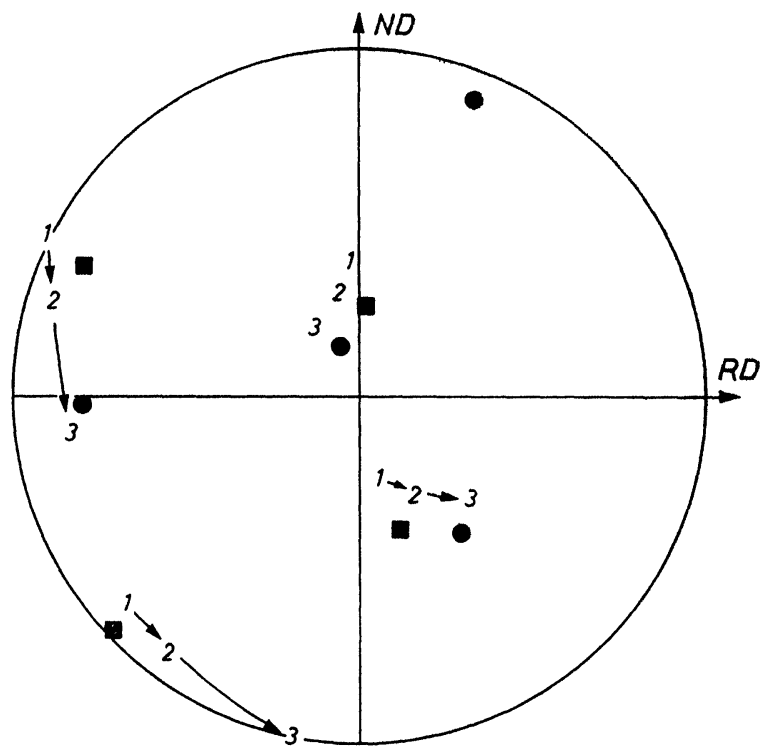

FIGURE 14 Transition from the surface texture component $A(1)$ to the texture of the interior of the material (O) in stereographic projection ((111)-poles; see text).

\section{DISCUSSION}

The question, to what extent transmission pictures yield, at least qualitatively, information on the arrangement and orientation of crystallites in polycrystalline material, has already been discussed repeatedly. The essential prerequisite for such an approach is that the boundaries between regions of different orientations be recognizable. In heavily deformed materials, these boundaries can be concealed by contrasts due to lattice defects, especially dislocation tangles, and to irregular extinction contours. It is, therefore, not surprising that the transmission pictures of strongly deformed materials frequently seem to indicate boundaries, whereas the diffraction pattern yields the same orientation on either side of the boundary, and vice versa. In other words, the structures visible on transmission pictures do not, in general, lead to reliable information on the course of boundaries between two areas of uniform orientation. This difficulty becomes particularly evident when comparing the strikingly similar transmission pictures of poly- and singlecrystalline materials, cf. Figures 9 and 13. Another example is the complete absence of a distinct boundary between the surface component $B$ and the matrix orientation of the deformed single crystal in the transmission picture (cf. Figures 12a and 13). Without making any arbitrary assump- 
tions, information on the position, size and shape of "crystallites" can only be obtained from the arrangement of the different areas of uniform orientations.

The cells in the transmission pictures which have already been observed and evaluated in the same cross-sections by $\mathrm{Hu}^{14,15}$ obviously differ in their significance from the regions of uniform orientation which were found in this work. According to $\mathrm{Hu}$, the mean thickness of the cells parallel to ND is $0.13 \mu \mathrm{m}$ which is practically the same as the values observed here. In contrast, the thickness of our regions of uniform orientation is about 4 to 8 times larger, i.e. 0.5 to $1 \mu \mathrm{m}$. Comparing this thickness with the size of the crystallites before rolling (10 to $15 \mu \mathrm{m}$ ), one recognizes immediately that this ratio of thickness correlates fairly well with the macroscopic reduction of the cross-section by rolling. This indicates that the regions of uniform orientation correspond to the original crystallite regions before rolling. The observation that no corresponding elongation of the crystallites in $\mathrm{RD}$ was found may be due to the fact that necking is more pronounced in general during tensile plastic deformation than in compression.

Another interpretation of the observed structure is suggested by our understanding of the formation of deformation band. ${ }^{24}$ Deformation bands are lattice regions of different orientations which are formed under conditions of multiaxial stresses during deformation. ${ }^{25}$ The original crystallites are thus divided as a result of the operation of different glide systems. The misorientation between the deformation bands increases with increasing deformation, until stable orientations with several mutually symmetrical components are finally formed. ${ }^{26,27}$ In the case of rolled f.c.c. metals, the two components of the (110)[112] orientation are such stable final orientations. It is only with these two mean preferential orientations that regions of uniform orientation very frequently have common boundaries (complementary orientations; compare 三 and IIII in Figure 3). Such complementary orientations with common boundaries could be observed clearly in the rolling plane but not in the other two cross-sections. Therefore, it can be assumed that many of the boundaries perpendicular to the rolling plane between the regions of uniform orientation have been formed by a process similar to the formation of deformation bands. In this model, too, the boundaries parallel to the rolling plane correspond to the original boundaries of the crystallites.
The fine structure within the regions of uniform orientation was not investigated. However, $\mathrm{Hu}^{15}$ has interpreted the cells visible in the transmission pictures as subgrains of slight mutual misorientation. For this reason, the rolled polycrystalline structure may be characterized crudely as follows: it consists of regions of uniform orientation having the shape of discs. The disc plane lies parallel to the rolling plane. Within such a region of uniform orientation, narrow elongated cells which may be considered as mutually misoriented subgrains extend in the rolling and transverse directions.

In regard to the recrystallization of such a material, two conclusions may be drawn:

1) The formation of a large angle boundary, ${ }^{28}$ be it by subgrain coalescence ${ }^{26,29}$ or by subgrain growth $^{\mathbf{3 0 - 3 2}}$ is possible, in a statistical sense, within areas having the thickness of the regions of uniform orientation, i.e. in this case within a dimension of 0.5 to $1 \mu \mathrm{m}$. As an alternative concept of nucleation, the bulging mechanism ${ }^{33,34}$ consists of the sharpening of the boundaries between regions of uniform orientation and the subsequent local bulging of some boundaries.

2) The energy stored in the material in the boundaries separating regions of uniform orientation and within the subgrain boundaries between the cells can be estimated, if the morphology and the mean misorientations are known. With the assumptions that the specific interfacial energy of the regions of uniform orientation is, on the average, $500 \mathrm{erg} / \mathrm{cm}^{2}$ and that the misorientations between the individual cells vary statistically, one obtains for the material under consideration a free energy of $3 \times 10^{7} \mathrm{erg} / \mathrm{cm}^{3}$. This is in order-of-magnitude agreement with the values obtained by calorimetry, which is reasonable.

In conclusion, considering the regions of uniform orientation, a critical remark on their size shall be made. Two crystallites, whose orientations are crystallographically equivalent (point-symmetric), cannot be distinguished by electron microscope, as mentioned in an earlier footnote. If identical diffraction patterns are observed in a grid region, this does not necessarily mean that one single orientation prevailed in this region. It is theoretically conceivable, though most improbable, that this region consisted of two crystallographically equivalent orientations. It is thus possible that the structure we have investigated contains, on the statistical average, more boundaries than indicated in the topographical representation. 
In earlier investigations of the orientation distribution of the crystallites in the same polycrystalline copper rolled $95 \%$, both the geometrically obvious analysis ${ }^{3}$ and the mathematically exact analysis ${ }^{7}$ of the data yielded concentrations of the crystallite orientations around (123)[745] and (112)[645]. The observation that single crystals of these two orientations hardly exhibit any change in orientation after rolling to $95 \%$ reduction is in agreement with these earlier results.

Under conditions of high friction between metal and rolls, a special texture has been found at the rolling surface of polycrystalline copper, namely the (100)[011] type. ${ }^{35,36}$ Apart from the [100][011] surface texture, components with (111) planes parallel to the surface have been observed in aluminium. ${ }^{35,36}$ It is remarkable that in the present work such a surface texture, namely the component $\mathrm{B}$, has also been found in copper. All these textures are essentially shear textures. ${ }^{35,36}$ The observation that these surface textures also develop in the three single crystals of different orientations corroborates the shear hypothesis.

It is obvious that the transition from the surface texture to the interior texture can occur in two ways: the transition of the component $\mathrm{A}$ is gradual (Figure 14), whereas in the case of the component $B$ a distinct boundary develops with respect to the matrix orientation (Figure 12). It is remarkable that both surface texture components can be transformed into the matrix orientation by rotation around an axis close to TD (cf. Figure 11a). Another striking feature is that, in all three surface texture components observed by X-rays, relatively closely packed planes lie parallel to the rolling plane, a cube plane in the case of components A and $C$, and an octahedral plane in the case of component $\mathrm{B}$. Thus the question whether this is valid for all surface textures remains to be answered.

The foregoing discussion dealt essentially with the conclusions drawn more or less directly from the observations. The additional information contained in the topographic correlations between the regions of uniform orientation will be treated in a separate publication.

\section{REFERENCES}

1. F. Haeßner, U. Jakubowski and M. Wilkens, Phys. Stat. Sol. 7, 701 (1964).

2. K. Lücke, H. Perlwitz and W. Pitsch, Phys. Stat. Sol. 7, 733 (1964).
3. F. Haeßner, U. Jakubowski and M. Wilkens, Mater. Sci. Engng. 1, 30 (1966).

4. H. Perlwitz, W. Pitsch and K. Lücke, in Recrystallization, Grain Growth and Textures (American Society for Metals, Metals Park, Ohio, 1966), p. 367.

5. F. Haeßner and W. Hemminger, Z. Metallkde. 58, 104 (1967).

6. F. Haeßner and D. Keil, Z. Metallkde. 58, 220 (1967).

7. H. J. Bunge and F. Haeßner, J. Appl. Phys. 39, 5503 (1968).

8. H. Perlwitz, K. Lücke and W. Pitsch, Acta met. 17, 1183 (1969).

9. H. J. Bunge, Mathematische Methoden der Texturanalyse (Akademie-Verlag, Berlin, 1969).

10. J. S. Kallend and G. J. Davies, J. Inst. Metals 99, 257 (1971).

11. H. J. Bunge, to be published in the Proc. Intern. Seminar: Quantitiative Analysis of Textures, Krakow, 1-6 September, 1971.

12. F. Haeßner, U. Jakubowski and M. Wilkens, Acta met. 14, 454 (1966).

13. R. C. Glenn, G. Langford, and A. S. Keh, Trans. ASM 62, 285 (1969).

14. H. Hu, Z. Metallkde. 60, 69 (1969).

15. $\mathrm{H}$. Hu, in Textures in Research and Practice, J. Grewen and G. Wassermann, eds. (Springer-Verlag, Berlin, 1969), p. 200.

16. G. Hoschek, Master thesis, University Stuttgart (1969).

17. W. Pitsch, H. J. Perlwitz, and K. Lücke, Z. Metallkde. 61, 231 (1970).

18. B. J. Duggan and R. L. Segall, Acta met. 19, 317 (1971).

19. W. Riecke, Optik 18, 278 (1961).

20. R. Gotthardt, Master thesis, University Stuttgart (1967).

21. G. Wassermann and J. Grewen, Texturen metallischer Werkstoffe (Springer-Verlag, Berlin, 1962), p. 58.

22. F. Haeßner, in Textures in Research and Practice, J. Grewen and G. Wassermann eds. (Springer-Verlag, Berlin, 1969), p. 1.

23. P. W. Bridgeman, Studies in Large Plastic Flow and Fracture (Harvard University Press, Cambridge, 1964).

24. W. Heye and H. P. Sattler, Z. Metallkde. 62, 386 (1971).

25. C. S. Barrett, in Cold Working of Metals (American Society for Metals, 1949), p. 65.

26. $\mathrm{H}$. $\mathrm{Hu}$, in Recovery and Recrystallization of Metals, L. Himmel ed. (Interscience Publishers, New York, 1963), p. 311.

27. H. Ahlborn, Z. Metallkde. 56, 205 (1965); 56, 411 (1965) and 57, 877 (1966).

28. R. W. Cahn, in Recrystallization of Metallic Materials, F. Haeßner ed. (Dr. Riederer-Verlag, Stuttgart, 1971), p. 43.

29. H. Hu, Trans. Met. Soc. AIME 224, 75 (1962).

30. W. G. Burgers, Rekristallisation, Verformter Zustand und Erholung (Akademische Verlagsgesellschaft, Leipzig, 1941).

31. R. W. Cahn, Proc. Phys. Soc (London) 63A, 323 (1950).

32. J. L. Walter and E. F. Koch, Acta Met. 10, 1059 (1962).

33. P. A. Beck and P. R. Sperry, J. Appl. Phys. 21, 150 (1950).

34. J. E. Bailey and P. B. Hirsch, Proc. Roy. Soc. 267A, 11 (1962).

35. P. Regenet and H. P. Stüwe, Z. Metallkde. 54, 273 (1963).

36. I. L. Dillamore and W. T. Roberts, J. Inst. Metals 92 , $193(1963 / 4)$. 